\title{
Run by Run Control of Chemical-Mechanical Polishing
}

\author{
Duane S. Boning, Member, IEEE, William P. Moyne, Taber H. Smith, Member, IEEE, \\ James Moyne, Roland Telfeyan, Arnon Hurwitz, Scott Shellman, and John Taylor
}

\begin{abstract}
A prototype hardware/software system has been developed and applied to the control of single wafer chemicalmechanical polishing (CMP) processes. The control methodology consists of experimental design to build response surface and linearized control models of the process, and the use of feedback control to change recipe parameters (machine settings) on a lot by lot basis. Acceptable regression models for a single wafer polishing tool and process were constructed for average removal rate and nonuniformity which are calculated based on film thickness measurement at nine points on 8 in blanket oxide wafers. For control, an exponentially weighted moving average model adaptation strategy was used, coupled to multivariate recipe generation incorporating user weights on the inputs and outputs, bounds on the input ranges, and discrete quantization in the machine settings. We found that this strategy successfully compensated for substantial drift in the uncontrolled tool's removal rate. It was also found that the equipment model generated during the experimental design was surprisingly robust; the same model was effective across more than one CMP tool, and over a several month period. We believe that the same methodology is applicable to patterned oxide wafers; work is in progress to demonstrate patterned wafer control, to improve the control, communication, and diagnosis components of the system, and to integrate real-time information into the run by run control of the process.
\end{abstract}

Index Terms-CMP, GCC, planarization.

\section{INTRODUCTION}

$\mathbf{T}$ HE chemical-mechanical polishing (CMP) process is of critical importance to current and future generation interconnect for integrated circuit technologies. In addition to CMP process and equipment development [1], the modeling of CMP processes is an active area of research, including work on wafer scale dependencies [2], feature scale models [3], as well as behavior of the equipment over many runs [4], [5]. The challenges posed by CMP for both sensor and control research are also becoming better known [6], [7]. While a good deal of research into run by run control methods has been reported

Manuscript received May 9, 1996; revised November 23, 1996. This paper was supported in part by SEMATECH, by ARPA under Contract NC001-0014, and by the Semiconductor Research Corporation under Contract 94-YC-085.

D. S Boning, W. P. Moyne, and T. H. Smith are with the Microsystems Technology Laboratories, Massachusetts Institute of Technology, Cambridge, MA 02139 USA.

J. Moyne and R. Telfeyan are with the University of Michigan, Center for Display Technology and Manufacturing, University of Michigan, Ann Arbor, MI 48109 USA.

A. Hurwitz and S. Shellman are with Statistical Methods and Advanced Process Control, SEMATECH, Austin, TX 78741 USA.

J. Taylor is with Compugenesis, Inc., Austin, TX 78739 USA.

Publisher Item Identifier S 1083-4400(96)09575-7. (see [8] for a review), relatively little practical experience with CMP control exists [7], [9], [10]. In this paper, we present the application of an integrated hardware/software control system utilizing run by run methods to overcome common CMP process and equipment difficulties.

In Section II, we briefly review CMP process and equipment fundamentals, and identify the difficulties accommodated through run by run process control. Section III presents the overall control system architecture, and describes the gradual mode run by run control strategy. The polynomial response surface and linear control models developed for CMP are discussed in Section IV. In Section V, we present a pair of simulation and fabrication experiments that demonstrate the importance and effectiveness of model adaptive run by run control. Finally, in Section VI we draw conclusions based on these experiments, and highlight areas where additional research and demonstration are needed.

\section{THE CMP PROCESS}

In the CMP process, the wafer is affixed to a wafer carrier (via back-pressure), and pressed face-down on a rotating platen holding a polishing pad, as illustrated in Fig. 1. A slurry with abrasive material (e.g., silica particles of sizes from $10 \mathrm{~nm}-200$ $\mu \mathrm{m})$ held in suspension is dripped onto the rotating platen during polish. The carrier and platen rotate at variable speeds, typically on the order of $30 \mathrm{rpm}$. Tools differ in the number of wafers that may be simultaneously polished; single-wafer, dual-wafer, and other multi-headed tools exist.

The process removes material at the surface of the wafer through a combination of mechanical and chemical action. A typical process goal is to achieve "global" planarization (across tens of $\mathrm{mm}$ ) by preferential removal of "high" material on the wafer. The planarization of dielectric (silicon dioxide) layers between multilevel metallization steps is one common application. Metal planarization is also often performed.

The control of CMP is chronically poor, arising from poor understanding of the process, degradation (wear-out) of polishing pads, inconsistency of the slurry, variation in pad physical properties, and the lack of in-situ sensors. Because the process includes mechanical abrasion of the surface, the polishing pad wears rapidly. Concurrent or sequential "conditioning" is usually employed whereby the abrasive surface of the pad is restored (either by mechanical damage to the surface or removal of a thin surface layer), but the lifetime of a pad remains on the order of a few hundred wafers. In addition to difficulties achieving a reliable film thickness (because of 


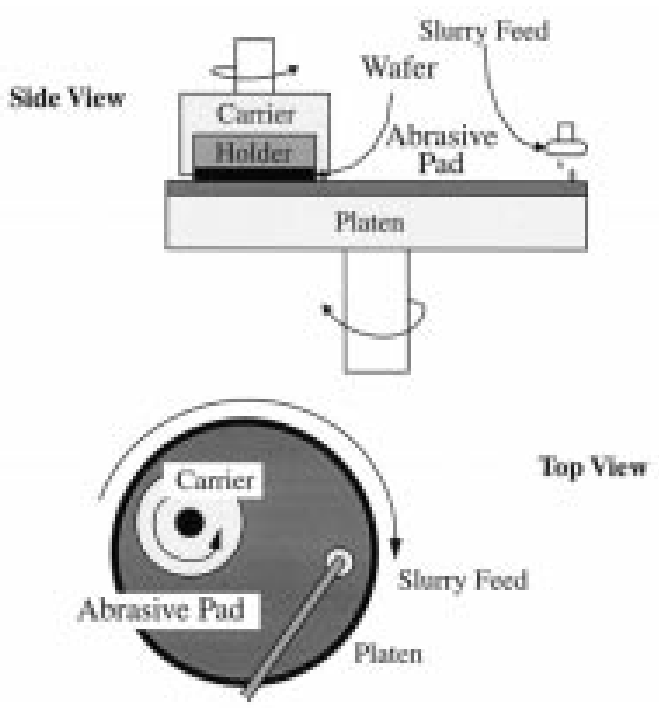

Fig. 1. Chemical-mechanical polish tool configuration.

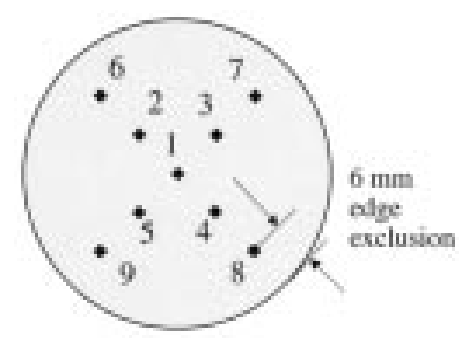

Fig. 2. Measurement sites.

changing removal rates over time), the within-wafer uniformity of the polish is difficult to achieve and maintain. Differences between polish rates at the center and edge of the wafer (i.e., "bulls-eye patterns") may arise due to wafer asymmetry (e.g., wafer flat), nonconstant relative pad velocity from the edge to the center, nonuniform slurry and by-product transport under the wafer, wafer bowing due to pressure, or machine drift in time of any of these parameters. As a result of these problems, it is conventional practice to use a number of send-ahead or dummy wafers to condition and/or calibrate the tool before or after each lot of wafers. Here we seek to employ run by run process control to address these process and tool issues-to both reduce or eliminate monitor wafer usage, and to maintain the performance of CMP processes.

In this work, the product characteristics of concern are the removal rate (corresponding to a controlled amount of oxide polished during the step) and the within-wafer uniformity of that removal rate across the wafer. The removal rate is determined by measurement of oxide film thickness before and after polish at each of nine sites on the wafer as shown in Fig. 2, divided by the (fixed) polish time. The "removal rate" output is the average of the nine sites on a wafer. The "nonuniformity" output parameter is computed for each wafer as the standard deviation of the amount removed over the nine sites on the wafer, divided by the average amount removed over the nine sites, times 100 .

Two sets of control experiments (detailed in Section V) were conducted, each on a different polisher. For each ex-
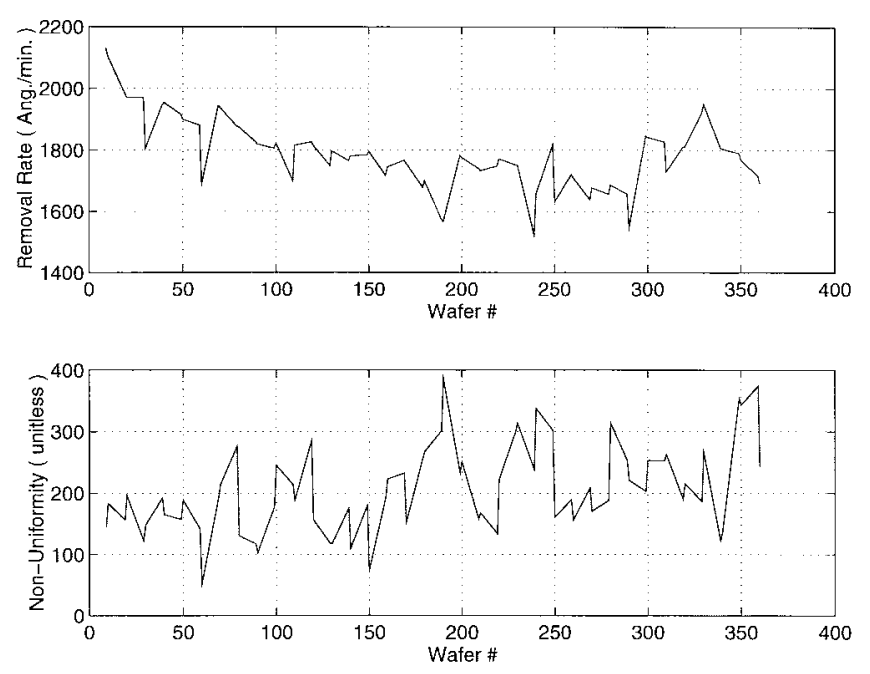

Fig. 3. Baseline CMP experiment.

periment, conventional slurry compositions, composite pads, and carrier films were used. Pad conditioning was performed simultaneously or sequentially, depending on the tools used. Film thicknesses were measured on a Prometrix Spectromap SM2000. In both experiments, the control goal is to maintain a target removal rate and within-wafer nonuniformity in the face of equipment drifts (primarily pad related) and disturbances. The change in removal rate and nonuniformity for a typical uncontrolled or baseline oxide polish process (with a fixed recipe) is shown in Fig. 3. This run, and all those described in this paper, were conducted on single wafer polishing tools, using 8 in silicon wafers with a thick blanket oxide deposition. Because we are only measuring and compensating for waferlevel variation from run to run, we believe that the same methodology is also applicable to patterned wafers in which similar wafer-level variation is typically observed [11].

\section{CONTROL System Description}

The observed drift in CMP processes, and the availability of post-process measurements, motivate the use of a run by run control strategy. A generic semiconductor control system framework has been under development [12]-[15], and is applied to the CMP control problem. Components of the control system design include the following.

1) Generic cell control-a framework for the implementation and incorporation of specific modules (e.g., for run by run control, communications, metrology, etc.). Generic services provided by the GCC include user interface, database access, and application module communication.

2) SECS-II communication with measurement and fabrication equipment. This enables capture of post-process wafer measurements, and of real-time equipment signals, as well as down-load of modified process recipes.

3) Statistical process control-conventional and multivariate charting, and mechanisms to dispatch to rapid or gradual mode controllers based on equipment state. 


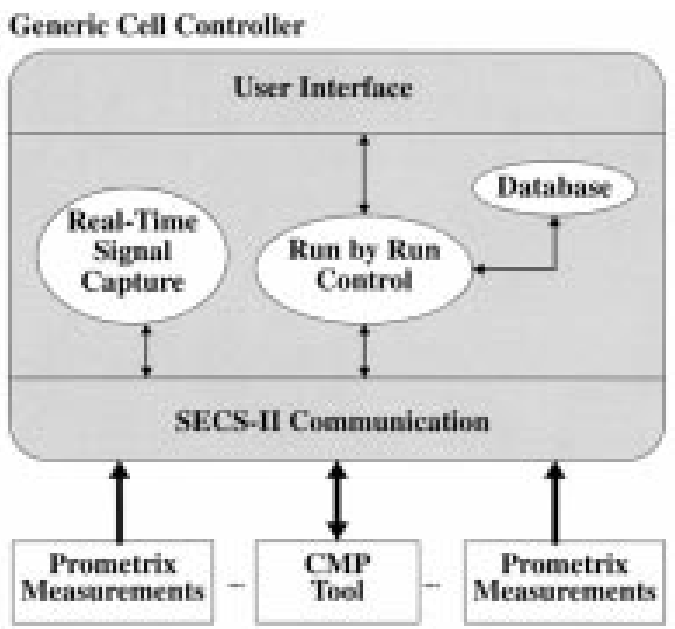

Fig. 4. Control system architecture with implemented components.

4) Rapid process adjustment-for rapid update of existing recipes (e.g., in response to significant shifts in equipment response), or rapid process evolution and optimization.

5) Run by run control-for gradual compensation, on a lot-by-lot basis, of equipment and consumables drift

In this paper, we focus on a subset of these modules used to demonstrate process control for CMP, as illustrated in Fig. 4. The collection of real-time data and preliminary data compression and diagnosis approaches (e.g., similar to that for plasma etch [16]) will be reported at a future time. A detailed discussion of the generic cell controller (GCC) is available in Moyne et al. [12]-[15]. A general introduction to run by run control is presented by Sachs [17]; the implementation and extensions of run by run control algorithms used here for CMP control are described in [10], and by Boning et al. [18].

The control architecture of Fig. 4 is expanded in Fig. 5 to highlight the control strategy used in this work. Offline experiments are performed on the CMP tool to build empirical (static input-output) models of the process response. An optimal process recipe is selected that satisfies (or trades off) design goals; this is used as the initial recipe for process control. Lots of 10 wafers each are planarized in the tool, and measurements of oxide film removal rate and nonuniformity are made on wafers \#9 and \#10. This information is fed to the gradual mode run by run controller, which adapts the process response models. These updated models are then used to generate a new process recipe which: a) achieves the best (weighted) trade-off among the multiple output targets or b) achieves all targets with the smallest (weighted) change in the recipe. The revised recipe is then used for the next lot of wafers.

\section{CONTROL Model DEVElopment}

Screening experiments with a set of seven parameters were initially performed to determine which machine parameters exert the strongest influence on removal rate and nonuniformity. Based on this initial screening, an experimental design for control purposes was restricted to speed, pressure, force,

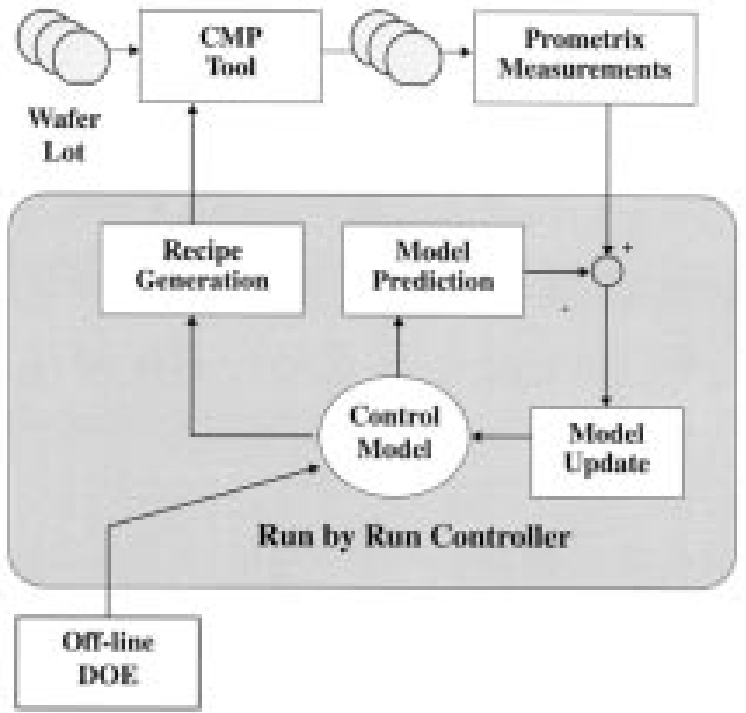

Fig. 5. CMP run by run control strategy.

TABLE I

EXPERIMENTAL DESIGN PARAMETERS

\begin{tabular}{c|c|c}
\hline Factor & Lower Bound & Upper Bound \\
\hline speed (rpm) & 20 & 40 \\
pressure (psi) & 0 & 7 \\
force (lb) & 8 & 10 \\
profile & -0.9 & 0.9 \\
\hline
\end{tabular}

and profile. A central composite design in these parameters was performed, with ranges as summarized in Table I.

Second order polynomial regression models were constructed for removal rate and nonuniformity. Model fits with adjusted $R^{2}$ of 0.897 and 0.769 for removal rate and nonuniformity, respectively, were achieved. The response surfaces (for nominal values of pressure and profile) are shown in Fig. 6.

Each polynomial regression model is linearized around the operating point to generate a multivariate model for the gradual mode run by run controller

$$
y=A x+c
$$

where $y$ is the output vector (removal rate and nonuniformity), $x$ is the input recipe vector (speed, force, pressure, and pad profile), $A$ is a $4 \times 2$ matrix of model coefficients, and $c$ is a vector of offset terms. In this controller, we only adapt or update the offset terms $c$, while the gain coefficients $A$ remain fixed. As seen in Fig. 6, the full response surfaces are nearly linear over the entire operating space. More importantly, the surfaces are locally very well-behaved (nearly linear, monotonic) thus justifying a linearization of the models for control. The robustness of these models in the face of equipment change is discussed in Section V-C.

\section{CONTROller Simulations AND EXPERIMENTS}

An important aspect of the run by run controller is adaptiveness of the process model. This is illustrated by comparison of two sets of control experiments: in the first, we use a "partial" 

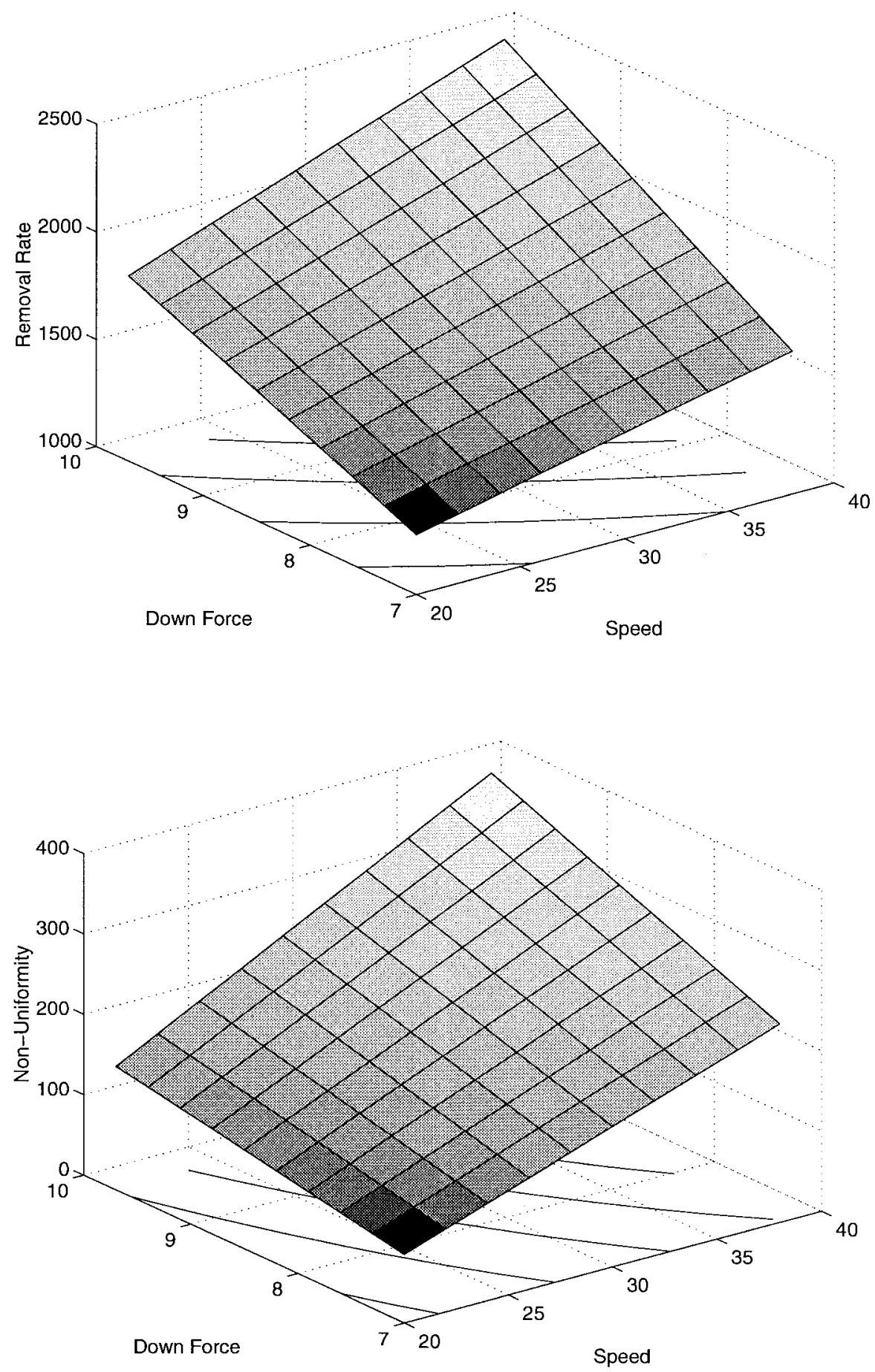

Fig. 6. Response surfaces for removal rate and uniformity (as a function of speed and force).

model update, and in the second we engage the full adaptive controller.

For partial model update, the linear model is updated based on the most recent run, but then combined with the original linear model before generating a new recipe

$$
c_{t}=\alpha\left(y_{t}-A x_{t}\right)+(1-\alpha) c_{0}
$$

where the subscript $t$ indicates the (lot) run number, and $y_{t}$ is the actual output resulting from the recipe $x_{t}$. The variable $c_{0}$ is the offset term in the original linear model. In this case, a weight coefficient $\alpha$ of 0.5 was used for removal rate, and 0.3 for nonuniformity. Under this strategy, the system functions similar to a discrete proportional controller (without integral action).

In the case of full model update, recursive adaptation occurs by an exponentially weighted moving average (EWMA) update of the offset term, again based on the error between model prediction and measurement

$$
c_{t}=\alpha\left(y_{t}-A x_{t}\right)+(1-\alpha) c_{t-1} .
$$



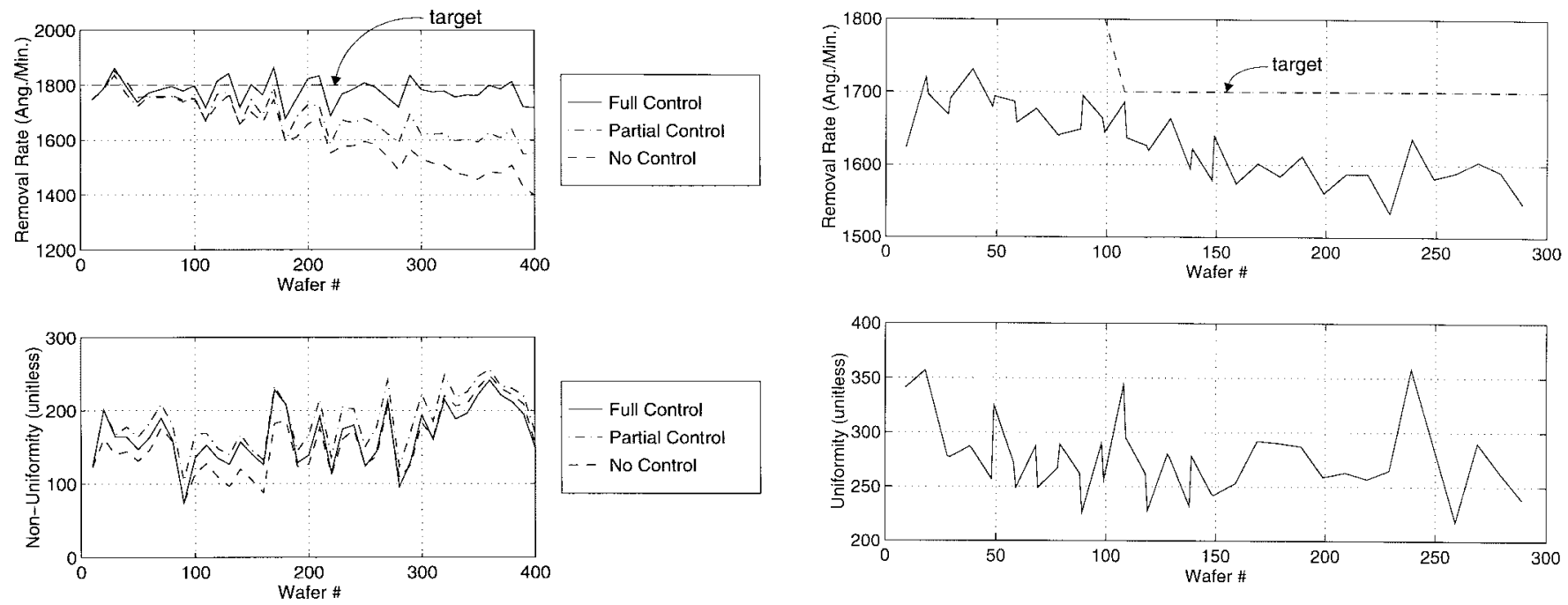

Fig. 7. Partial and full model update—output comparison (simulation).
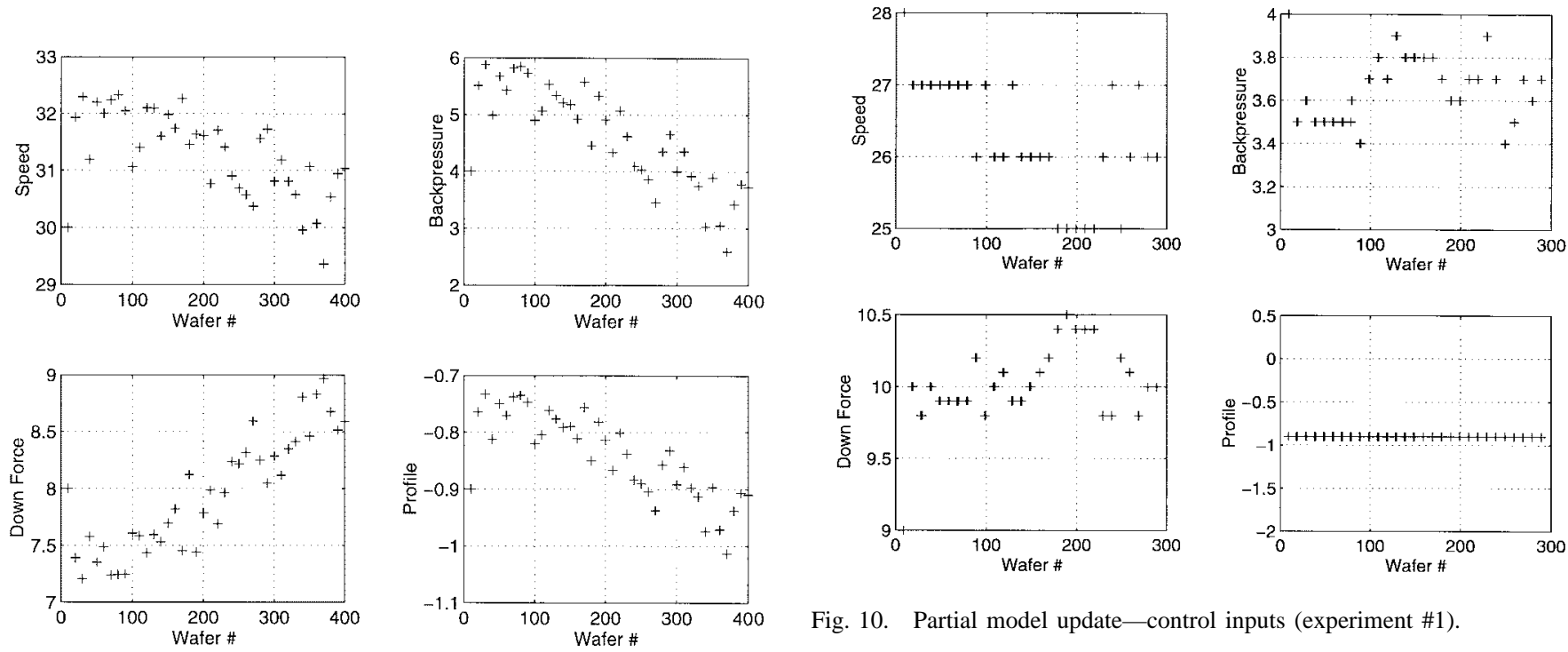

Fig. 10. Partial model update—control inputs (experiment \#1).

Fig. 8. Full model update—control inputs (simulation).

Here, $c_{t-1}$ is the offset term used on the previous run. The selection of the weight coefficient $\alpha$ is based on consideration of both noise and sampling (i.e., we measure and average only wafers 9 and 10 out of each 10 wafer lot). Again, weight coefficients of $\alpha=0.5$ for removal rate and 0.3 for nonuniformity were used.

Simulations were performed for these two cases, as shown in Figs. 7 and 8. An equipment simulation model was assumed with gain coefficients $5 \%$ greater or smaller than in the control model, linear drift on the removal rate and nonuniformity, and additive gaussian noise similar to those observed on the baseline run. We see that the partial model update does not adequately compensate for the drift in removal rate, although it does reduce the impact of the drift compared to an uncontrolled process. The full model update, on the other hand, succeeds in removing the drift, with a small bias error due to drift trend and model mismatch as expected [19].

The recipe (also called the machine settings, inputs, or control parameters) suggested by the controller are shown

in Fig. 8 for the full model update simulation. We see that full multivariate control action is taking place: all four input parameters are used by the controller to keep removal rate and uniformity on target. In these simulations and experiments, a low nonuniformity target (on the order of 100-200) below that typically achievable was used to keep the nonuniformity below some acceptable value (a future desirable enhancement to the controller is the inclusion of constraints on outputs in addition to or instead of specific target values). Note that in these simulations we assume very fine resolution on input parameters. In the experiments that follow, we incorporate extensions in the algorithm that explicitly account for finite input resolution (or quantization), observable as discrete jumps in the input parameters of Figs. 10 and 12. The algorithms used, including the utilization of input and output weights and constraints as well as input quantization, are described in detail in [18].

\section{A. Experiment \#1: Partial Model Update}

The results of the first control experiment, with only partial model update, are summarized in Figs. 9 and 10. Examining 

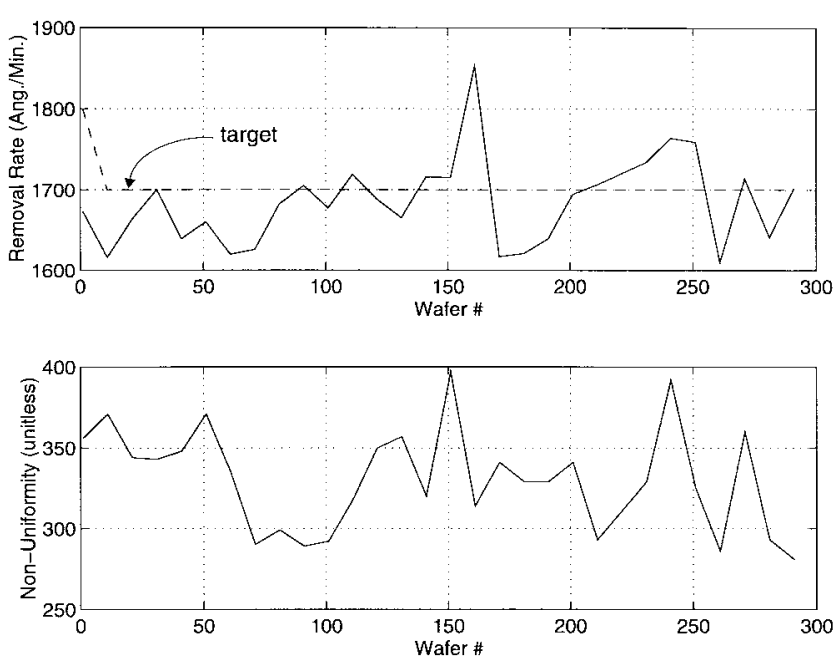

Fig. 11. Full model update-outputs (experiment \#2).

the output result for removal rate, we note that the original

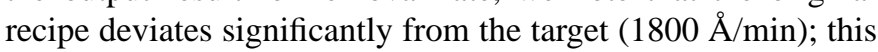
model error is fatal without permanent model adaptation. On each run, the controller generates a new recipe which attempts to fully compensate for the difference between desired and observed removal rate. This change initially (first 50 wafers or so) moves us closer to the target, but once substantial drift accumulates, the controller has no means to permanently improve the control-on each new run, the controller generates a new recipe which is an improvement over the original recipe, but not an improvement over the previous recipe. The controller does not take advantage of the full range of input parameters (they have not hit bound constraints); rather, the problem is that no permanent adaptation or learning is achieved, and the resulting control is poor.

\section{B. Full Model Update Experiment}

In the second experiment, full (permanent) model adaptation was used. Here we see (Fig. 11) that the drift is compensated much more effectively. Examining the control parameter moves in Fig. 12, we find that the algorithm responds well, producing successively more aggressive control effort to compensate for accumulating drift. At the same time as speed is increased to maintain removal rate, however, we also see that force is decreased to improve uniformity. The trade-off between these two goals (seen in the response surface of Fig. 6) is also apparent in the resulting control action.

We can compare the baseline, partial model update, and full model update experiments in terms of mean square error (mean deviation from target squared) in the removal rate. We find that the baseline gives MSE of $3.2 \times 10^{4}$, partial model update of 129, and full model update 36 . We thus see that the full model update strategy performs substantially better than no control or the partial model update strategy.

\section{Control Model Robustness}

An interesting issue is the robustness and stability of the regression linearization models used for control [19]. The
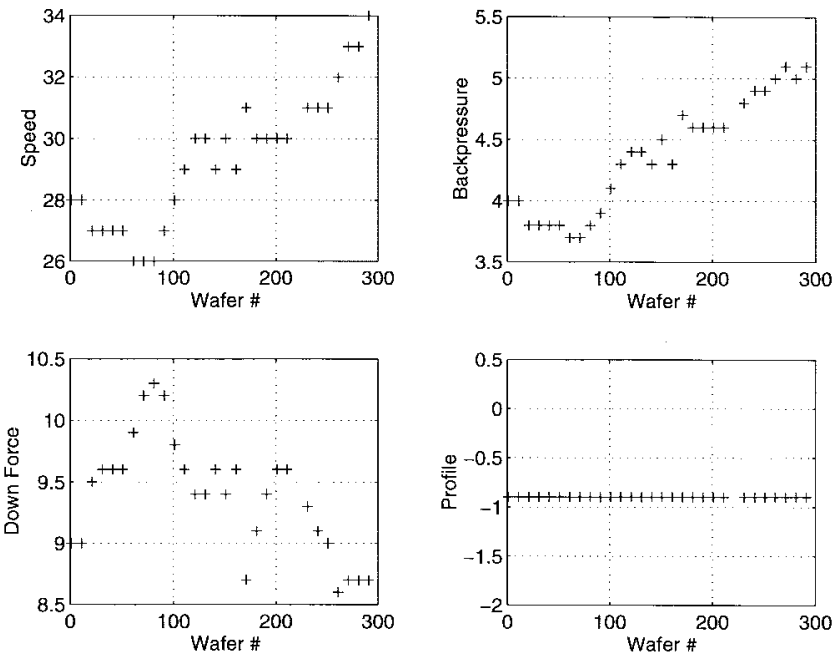

Fig. 12. Full model update-control inputs (experiment \#2).

original regression model and its linearization (Section IV) formed the basis for the first set of control experiments. These control models were in fact developed on a different tool than that used for the control experiments, and were developed several months earlier. We found that the CMP control models appear to capture fundamental aspects of CMP (e.g., removal rate increases with increased polish speed and force, as expected). The error in model gain coefficients (e.g., the gain with respect to polish speed) does, however, contribute to the bias error observed in controlled outputs. Further work to address this bias is underway (i.e., predictorcorrector approaches to attack persistent trends [20], internal model control for better convergence [21]).

To more directly assess the validity of the control models across time and tool type, upon completion of the first control run, a small experimental design was again performed, this time directly on the tool used for the control experiment. A 40 wafer Box-Behnken design in the four control parameters was performed. The resulting models were found to be surprisingly similar to the original models; some coefficients (e.g., speed term in the removal rate model) were numerically very similar, and the remaining coefficients were all of the same sign as the control models. These modeling and control results are encouraging, and indicate that expensive recalibration of control models may not be necessary.

\section{CONCLUSION}

In this paper, we have demonstrated the successful application of run by run control to chemical-mechanical polishing. CMP processes pose significant problems: lack of endpoint or other in-situ sensors, poor (primarily empirical) process understanding, and chronic equipment drift. An integrated control system is under development to address these problems that incorporates run by run control algorithms, real-time monitoring to identify equipment problems, and a generic cell controller for equipment communication, data management, and control execution. In this paper, we have focused on run by run control demonstration utilizing post-process measurements, linearized response surface models from design 
of experiments, and gradual exponentially weighted moving average model adaptation and recipe adjustments to maintain process goals. We have also shown the importance of model adaptation for removal rate maintenance. By incorporating multivariate [multiple input multiple output (MIMO)] control, weighted constrained recipe generation, and quantization of inputs, we have demonstrated control of removal rate while maintaining adequate uniformity.

This work highlights several areas for future research. First, real-time monitoring by way of data compression (principal component analysis) in combination with function approximation offers the hope of 1) early detection and diagnosis of machine problems, and 2) correlation with machine state (e.g., drift) that can feed into better control of the process. Second, additional practical issues remain for full automation of the run by run controller, including rapid mode integration, the optional selection of a dead band (to reduce or trade-off the number of control moves if desired), and the support of output constraints (rather than requiring output targets). Third, the investigation of additional run by run control methods appears fruitful, potentially including classical LQG, neural network, or stochastic dynamic programming approaches that can take full advantage of emerging empirical and physical models of the process (particularly those incorporating process dynamics) [22], [23]. These experiments have been performed on blanket (unpatterned) oxide wafers; control of uniformity on patterned wafers is currently being pursued and will be presented in the future. Finally, the issues of control for metal and damascene CMP processes are also of practical and future interest and should be investigated.

\section{ACKNOWLEDGMENT}

The authors would like to thank IPEC/Planar for their assistance in these experiments.

\section{REFERENCES}

[1] M. Fury, "Emerging developments in CMP for semiconductor planarization," Solid State Technol., pp. 47-54, Apr. 1995.

[2] S. Runnels and L. Eyman, "Tribology analysis of chemical-mechanical polishing," J. Electrochem. Soc., vol. 141, no. 6, pp. 1698-1701, June, 1994.

[3] S. Runnels, "Feature-scale fluid-based erosion modeling for chemicalmechanical polishing," J. Electrochem. Soc., vol. 141, no. 7, pp. 1900-1904, July, 1994

[4] A. Hu, X. Zhang, E. Sachs, and P. Renteln, "Application of run by run controller to the chemical-mechanical planarization process, Part I," in Proc. IEEE 15th Int. Electron. Manufact. Technol. Symp., Oct. 1993.

[5] A. Hu, H. Du, S. Wong, P. Renteln, and E. Sachs, "Application of run by run controller to the chemical-mechanical planarization process, Part II," in Proc. IEEE 16th Int. Electron. Manufact. Technol. Symp., Oct. 1994.

[6] R. Jairath and L. Markert, "Metrology and process control issues in chemical mechanical polishing," in NIST 1995 Semiconductor Characterization Workshop, Jan. 1995.

[7] A. Hu, H.-P. Dun, P. Renteln, and E. Sachs, "Sensor development and process control for chemical-mechanical planarization of multilevel interconnect devices," in Electrochem. Soc. Meet., June 1995.

[8] E. Del Castillo and A. Hurwitz, "Run to run process control: A review and some extensions," J. Qual. Technol., Feb. 1994.

[9] A. Altman, "Applying run by run process control to chemicalmechanical polishing of sub-micron VLSI: A technological and economic case study," S.M. thesis, Department of Electrical Engineering and Computer Science, Massachusetts Institute of Technology, May 1995.
[10] W. Moyne, "Run by run control: Interfaces, implementation, and integration," S.M. thesis, Department of Electrical Engineering and Computer Science, Massachusetts Institute of Technology, May 1995.

[11] R. R. Divecha, B. E. Stine, D. O. Ouma, D. Boning, J. Chung, O. S. Nakagawa, S.-Y. Oh, and D. L. Hetherington, "Comparison of oxide planarization pattern dependencies between two different CMP tools using statistical metrology," in VLSI Multilevel Interconnect Conf., Santa Clara, CA, June 1996, pp. 427-430.

[12] J. Moyne and L. McAfee, Jr., "A generic cell controller for the automated VLSI manufacturing facility," IEEE Trans. Semiconduct. Manufact., vol. 5, pp. 77-87, May 1992.

[13] J. Moyne, H. Etemad, and M. Elta, "A run-to-run control framework for VLSI manufacturing," in Proc. Microelectron. Process., Sensors, Controls, SPIE, vol. 2091, 1994, pp. 379-390.

[14] R. Telfeyan, J. Moyne, A. Hurwitz, and J. Taylor, "Demonstration of a process-independent run-to-run controller," in Electrochem. Soc. Meet., June 1995.

[15] J. Moyne, R. Telfeyan, A. Hurwitz, and J. Taylor, "A Processindependent run-to-run controller and its application to chemicalmechanical planarization," in Proc. 6th Ann. SEMI/IEEE ASMC, Boston, Nov. 1995.

[16] D. White, "In-situ wafer uniformity estimation using principal component analysis methods," S.M. thesis, Department of Electrical Engineering and Computer Science, Massachusetts Institute of Technology, May 1995 .

[17] E. Sachs, A. Hu, and A. Ingolfsson, "Run by run process control: Combining SPC and feedback control," IEEE Trans. Semiconduct. Manufact., vol. 8, pp. 26-43, Feb. 1995.

[18] D. Boning et al., "Practical issues in run by run control," in Proc. 6th Ann. SEMI/IEEE ASMC, Boston, MA, Nov. 1995.

[19] A. Ingolfsson and E. Sachs, "Stability and sensitivity of an EWMA controller," J. Qual. Technol, vol. 25, no. 4, pp. 271-287, Oct. 1993.

[20] S. W. Butler and J. Stefani, "Application of predictor corrector control to polysilicon gate etching," in Proc. Amer. Control Conf., June 1993.

[21] M. Morari and E. Zafiriou, Robust Process Control. Englewood Cliffs, NJ: Prentice-Hall, 1989.

[22] E. Zafiriou, H. Chiou, and R. Adomaitis, "Nonlinear model based runto-run control for rapid thermal processing with unmeasured variable estimation," in Electrochem. Soc. Meet., June 1995.

[23] E. Zafirious, R. Adomaitis, and G. Gattu, "An approach to run-to-run control for rapid thermal processing," in Proc. Amer. Control Conf., 1995.

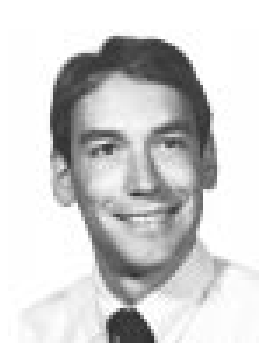

Duane S. Boning (S'83-M'91) received the S.B. degrees in electrical engineering and in computer science in 1984, and the S.M. and Ph.D. degrees in 1986 and 1991, respectively, all from the Massachusetts Institute of Technology, Cambridge, MA.

He was an NSF Fellow from 1984 to 1989 , and an Intel Graduate Fellow in 1990. From 1991 to 1993, he was a Member of the Technical Staff at the Texas Instruments Semiconductor Process and Device Center, Dallas, TX, where he worked on process/device simulation tool integration, semiconductor process representation, and statistical modeling and optimization. $\mathrm{He}$ is currently an Assistant Professor in the Electrical Engineering and Computer Science Department at MIT. His research focuses on variation modeling and control in semiconductor processes, with special emphasis on chemical-mechanical polishing and plasma etch. Additional interests include tools and frameworks for process and device design, network technology for distributed design and fabrication, and computer integrated manufacturing. $\mathrm{He}$ is an Associate Editor for the IEEE Transactions on SEMICONDUCtor MANUFACTURING.

Dr. Boning is a member of Eta Kappa Nu, Tau Beta Pi, Sigma Xi, and the Association of Computing Machinery.

William P. Moyne received the B.S.E. degree in electrical engineering and computer science from the University of Michigan, Dearborn, in 1992, the S.M. degree in run-by-run control at the Massachusetts Institute of Technology, Cambridge, in 1995, and is currently a Ph.D. candidate.

His research focuses on process synthesis for semiconductor and MEMS applications. He is also interested in the areas of system design and distributed collaborative computing systems.

Mr. Moyne is a member of Tau Beta Pi and Eta Kappa $\mathrm{Nu}$. 
Taber H. Smith (M'96) received the B.S. degree in electrical engineering from the Rochester Institute of Technology, Rochester, NY, in 1994, the M.S. degree from the Massachusetts Institute of Technology, Cambridge, in 1996, and is currently a Ph.D. student.

His principle research interests include the application of statistical and intelligent control methods to semiconductor process control.

Mr. Smith is a member of Tau Beta Pi and Phi Kappa Phi.

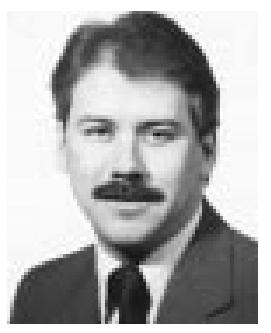

James Moyne received the B.S.E.E. and B.S.E. degrees in mathematics from the University of Michigan, Dearborn, in 1983, and the M.S.E.E. and Ph.D. degrees from the University of Michigan, Ann Arbor, in 1984 and 1990, respectively.

$\mathrm{He}$ is currently President of MiTeX Solutions, Inc., a supplier of complete run-to-run control solutions. He has a background in discrete control, networks, sensor busses, mathematics, and databases, and has a patent on a generic cell controller design.

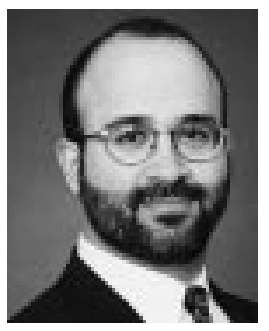

Roland Telfeyan received the B.S. degree in mathematics and the B.M. degree in music theory from Northwestern University, Evanston, IL, in 1985 and 1988, respectively, and the M.M. degree in music theory from the University of Michigan, Ann Arbor, in 1990.

From 1990 to 1992, his doctoral research in music theory included the study of complex objectoriented software systems. From 1993 to 1996, he was a Systems Research Programmer at the University of Michigan Department of Electrical Engineering and Computer Science, where he co-designed and developed a fully object-oriented implementation of the Generic Cell Controller. From 1990 to 1995, he founded and managed Telf Design Corporation, a developer of custom software systems for medical, transportation, and telecommunications companies. He is currently a Vice President at NationsBanc Capital Markets, Inc., where he leads a team developing software for proprietary trading systems.

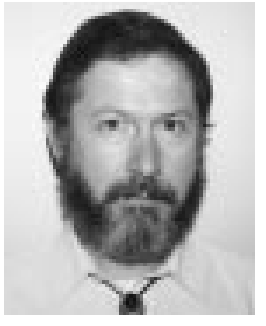

Arnon Hurwitz received the M.S. degree in applied statistics from Oxford University, U.K. and the $\mathrm{Ph} . \mathrm{D}$. degree in mathematical statistics from the University of Cape Town, South Africa.

$\mathrm{He}$ has been Manager at Sematech of Project J88D, a control project in the Advanced Process Control Group since January of 1994. Previously, he held posts in Statistical Methods at Sematech, and as Statistician at Corning Glass Inc., Telecommunications division, and at Himont USA (now Montel Inc.) the world's largest producer of bulk polypropylene.

Scott Shellman was born in Hood River, OR, in January 1970. He received the B.S. and M.S. degrees in statistics from Brigham Young University, Provo, UT.

He spent one term as an Intern Statistician for Sematech, Austin, TX, and two summers as an Intern Statistician for Micron in Boise, ID. His graduate research consisted of developing fault detection methods for real-time tool data in the presence of changing recipes. He is currently a Statistician for Intel Corporation, Aloha, OR.

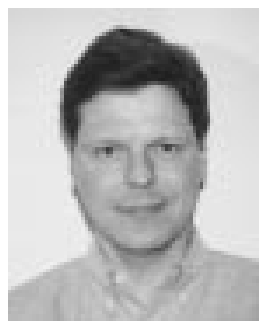

John Taylor received the B.A. degrees in economics and mathematics from Southern Methodist University, Dallas, TX.

In 1990, he founded Compugenesis, Inc. He has worked with Sematech, the Massachusetts Institute of Technology, Inc., and the University of Michigan, Ann Arbor, in the areas of multiple sensor integration and process control. Other work with Sematech and industry has included sensor prototype implementation, system integration, equipment communication, and data acquisition and control.

Prior to Compugenesis, he was a Senior Systems Analyst and Johnson Controls. He was involved in the design and implementation of distributed process control and SCADA Systems. 